\title{
Brouwer's Conception of Language, Mind and Mathematics ${ }^{1}$
}

\author{
Hiroshi KaneKo ${ }^{2}$
}

It has been the main stream of research into intuitionsim for some decades to try to interpret intuitionist criticism of classical mathematics from a constructive semantic point of view. In such attempts, however, it is impossible to include all intuitionistic tenets, especially Brouwer's criticism of language and communication, in such a semantic framework. Some parts of Brouwer's thoughts thoroughly resist any kind of semantic interpretation. This is similar to Wittgenstein's later philosophy which also does not fit any kind of semantic interpretation, or to interpretations that his philosophy is constructed to exclude any semantic readings. By this comparison I hope to suggest that we have something to learn from Brouwer's philosophy, especially his criticism of language and linguistic communication that resists any semantic readings ${ }^{3}$. In fact, Brouwer exerted influence on peoples such as Hilbert and Weyl for almost two dacades before the beginning of semantic research, that is, before the formulation of intuitionistic logic by Heyting. So, we can raise a legitimate question about what influence Brouwer exerted. Roughly speaking, I think it was his prior insights into the relation between language and mathematics. In the following, I would like to consider what those insights are, what kind of view on language they are based on and how they influence the foundational debates.

\section{Criticism against linguistic mathematics}

In his paper [1928], Brouwer claims that the formalist concept of metamathematics came originally from his conception of second-order mathematics. The idea of second-order mathematics first appears in his dissertation. In fact, Brouwer analyzes the content of Hilbert [1900] in detail there under the heading "Enumeration of the stages which are confused in the logical treatment of mathematics" and in the preceding paragraph he sums up what becomes evident from this analysis as follows.

${ }^{1}$ I am grateful to Michael Detlefsen for valuable comments on the earlier version of this paper. I am also grateful to Jeffrey Fryckman who helped me to correct grammatical mistakes. Possible remaining mistakes are my own.

${ }^{2}$ E-Mail: hkaneko@isc.senshu-u.ac.jp Department of Philosophy, Senshu University

${ }^{3}$ Although I say so, I do not intend to say that there is no dogmatic claim in his philosophy nor that all of his claims are understandable. 
The method which we reject here surpasses that of the logicians in that it takes, several times in succession, the unjustified step from the original mathematical domain to another by means of the linguistic act, and it does not, as the logicians do, maintain the intuitive connection between the two mathematical domains which are connected only through the intermediary of language ${ }^{4}$.

Here the method to be rejected is the formalist one (or the earliest form of the Hilbert-program). In Hilbert [1900] Hilbert proposes to axiomatize arithmetic. In doing so, he must presuppose an arithmetical domain which is to be axiomatized. It is this domain that is refered to as "the original mathematical domain". But in order to perform this project, the language which used to construct the axiom system must be conciously constructed in advance. According to Brouwer, we have already a problem at this stage. For we may not be able to succeed in connecting the thought which accompanies the construction with symbolic signs. This difficulty is shared by both logicists and formalists. But Hilbert will have another difficulty. For, according to his project, he must not only present the axiom system of real numbers, but also he must show that this axiom system is complete and consistent. Therefore, after he constructs the axiom system that describes the original mathematical domain, at the next stage he must objectify the axiom system itself and operate on this linguistic axiom system. For example, we may take a proof in this axiom system as an object of our investigation. Then, to begin with, there is a principle of complete induction among principles which support this proof-like linguistic construct as a genuine proof. Also there are many elements like "mehere, zwei, Fortsetzung, an Stelle von, beliebig, etc"5. here. Those principles and elements will constitute another mathematical domain. It is this domain that Brouwer called as second-order mathematics. But this is not an end of the story. In order to accomplish his project, Hilbert must eliminate the meaning from these proofs and mimic these proofs in still another domain(third-order mathematics) ${ }^{6}$. These two-step transitions from one domain to another is just what Brouwer called "the unjustified step".

Here I would like to note that we can divide difficulties which Brouwer pointed out to formalists and logicists into two different (but related) types. One type of difficulties is one that is shared by both formalists and logicists (indeed, we will further classify this one into two types). Another is an unjustified step which only formalists take. I will examine these two types of difficulties in detail.

It is well known that logicists have been criticized by Brouwer in their

${ }^{4}$ Brouwer [1907], p. 94.

5 ibid.

${ }^{6}$ Brouwer [1907] pp. 94-95. 
unlimited use of classical logic, especially in their application of tertium non datur to infinite domain. Why is this seen as a mistake? There are several ways to answer this question. One of them is to say that such logical principles are not reliable means to arrive at genuine mathematical results. Of course what I mean here as mathematical results is what we can get by means of constructive mathematical activities. That is, unlimited use of logical principles (typically applications of tertium non datur to infinite sets) might take us out to the place where constructive mathematical activities cannot reach. In other words, those logical principles make us overreach the boundaries of a constructive mathematical domain. Therefore, we cannot use classical logical principles in order to explain what mathematics is or to describe how we perform mathematical constructions. But this is only a part of the logicists mistake.

The logicians, conversely, start from these principles and as the foundamental domain of operations, within which the relation meant by the words or symbols must exist, they choose not some mathematical systems, but the chimerical 'everything' — as we saw above (p.138 seq. [p.78], footnote), Dedekind also wrongly tried to start from this notion — from which they define various class by what they call propositional functions ${ }^{7}$.

One of the characteristics of the logicist method is to deal not with mathematical proofs themselves but with their linguistic counterparts. This way of speaking may sound somewhat Brouwerian. In other words, logicists must prepare a formal language in advance for performing their project. Then they treat formal proofs as genuine mathematical proofs. In this case, manipulations of this language may produce extra entities which do not represent original mathematical entities or constructions because of the general purposes of the language. By saying that language serves general purposes we mean that objects which are linguistically constructed according to the grammar or legitimate principles are permitted as legitimate objects of the language without any constraints from a particular research field. Those ways of constructing objects are not necessarilly constrained only by mathematical principles. Brouwer realized a danger inherent in linguistic method here. This characteristic of logicist method invites in their domain extra constructs that do not correspond to any mathematical constructions, so logicists must presuppose the chimerical everything as their domain.

\footnotetext{
${ }^{7}$ Brouwer [1907] pp. 88.
} 


\section{An excessive productivity of language}

We can here sum up Brouwer's criticism of the logicist method as follows. First of all, classical logic which has been used by logicists is inappropriate for describing or explaining original (constructive) mathematical activities. It is not because logical principles are insufficient for describing mathematical activities but because these principles supply too much. For logical principles, which are used for organizing mathematical activities, may supply us the means for passing over the original mathematical domain ${ }^{8}$. Second, although we should start from actual mathematical constructions, logicist reverse the order and they start from linguistic principles and linguistic constructs. In consequence, they are compelled to presuppose a surplus domain which has no relation to inherent mathematical domain. Brouwer indicates that such a surplus domain is just a source of paradox ${ }^{9}$.

These critical arguments against logicism are based on Brouwer's recognition that linguistic methods provide mathematics with something which we cannot reach only by constructive mathematical activities ${ }^{10}$. As I called it previously, I would like to refer to such a power of language as an excessive productivity of language. However, errors by an excessive productivity of language are not restricted to those two.

According to Brouwer, if we see an axiom system itself as a linguistic construct, we can be in a position where we can take such a linguistic construct as an object of our assessment. Then we would apply logico-linguistic methods to such a construct again in order to establish the correctness of our assessment. It appears that such kind of actvity is possible because of the comprehensiveness of language. Here it is important to note that each mathematical domain which is presupposed by each mathematical activity performed in each stage must be different from each

${ }^{8}$ Classical logical principles supply too much because these principles are not principles that adhere to constructive mathematical activities but they are principles that hold only on 'concepts' and 'objects' which are linguistic objects. In such a sense, two errors of logicist's are closely related.

${ }^{9}$ Frege also arrives at the same point in analyzing a source of paradox and deplores that it is hard to controll language. See Frege [1925], p. 289.

${ }^{10}$ In Detlefsen[1990] similar points are already indicated. Detlefsen argues there that Brouwer rejected uses of logical principles in mathematics because the applications of logical principles did not preserve the qualities of mathematical recognition. This is an attempt to understand Brouwer's intuitionism as a kind of mathematical epistemology. This attempt is not opposed to my attempt to interpret Brouwer's criticism against language and communication as criticism of the excessive productivity of language. But my aim here is to make it clear that Brouwer's recognition of the conflict between applications of logical principles and mathematical epistemology is based on his recognition of the conflict between linguistic activities and mathematical activities and by doing that to bring his solipsist claims, criticism of language and communication and his intuitionism into an unified picture. 
other. Using Brouwerian terms, we cannot presuppose that the original mathematical domain would be the same as that of second order or third order. For if they are the same, our procedure of assesment would be circular. This circularity was already suggested by the Pincaré-Brouwer criticism of the principle of complete induction. So we must count these domains as different from each other. But, if they are different, how are we permitted to take a step from one domain to another? It seems that Brouwer insists that what make such transgressions possible are linguistic operations on linguistic descriptions of the domains. Here what I mean by linguistic operations are the operations like forgetting the senses of linguistic descriptions and assigning to them senses from another domain. In fact, Brouwer says, "Hilbert takes the step decisively and definitively and remains on the second level, returning to the first only to give it a sense in the second, then he takes a further step, again definitively, remains on the third level which he so creates, and uses the first and second only to give them a sense in the third"11. As I shall argue it later, I think Brouwer recognizes here that the premission of those linguistic operations presupposes a conception of language. Anyway, it is certain that Brouwer's criticism of Hilbert's unjustified steps shed light on a feature of linguistic acts. This feature is also counted as a kind of productivity of language because it makes possible the transition that we cannot conduct within intuitive mathematics. I would like to subsume this feature under the excessive productivity of language.

To sum up, what I mean by the excessive productivity of language is the following : (1) inferences that are based on classical logical principles take us to the position that we cannot reach in constructive mathematical activities. (Metaphorically speaking, classical logic is not a conservative extension of constructive mathematical activities.) (2) The linguistic method of concept formation invites the domain of everything into mathematics. (3) Language makes it possible to ascend to second and third order mathematics. This transition would be impossible without linguistic operations on linguistic constructs. When Brouwer criticized logicism and formalism, it was based on his recognition of these features of language.

\section{Brouwer's Solipsist Claims}

But here we can raise two questions about Brouwer's philosophy if solipsism is an essential feature of his thought. It is true that the most surprising features of Brouwer's philosophy are among his solipsist claims. But if these claimes are to the effect that linguistic communications are intrisically impossible, then where should we locate his criticism of logicists and formalists in his philosophy? For if it is claimed that any kind of linguistic communication is impossible, it seems that both

\footnotetext{
11 Brouwer [1907] p. 94.
} 
projects of logicists and formalists will be immediately rejected by Brouwer and that he need not conduct any further analysis and criticism of them.

Secondly, his recognition of the excessive productivity of language does not follow only from the position of viewing mathematics as constructive activities. If he insists only that mathematical activities must be constructive, the result will be the replacement of classical semantics by constructive semantics. But his criticism contains more than that. His claims based on the recognition of the excessive poductivity of language have some implications which makes the formalisation of intuitionistic logic and any kinds of semantic projects ineffective.

I think that in order to find answers to these questions we must understand the relation between Brouwer's solipsist claims and his recognition of the excessive productivity of language. So let us start to inquire into the solipsists claims. Here we have a sample list of his solipsist claims. I think such claims can be classified into a few groups.

The first group contains claims like :

in religious truth, i.e. in wisdom, which abolishes the discernment between the subject and something different, and where the perception of time is no longer admitted, there is no mathematical understanding, let alone reliability of $\operatorname{logic}^{12}$.

Although this quotation is only one example, Brouwer often describes in detail the stage where consciousness stays in its original state. We can see his basic claim about solipsism here.

The second group consists of claims like :

"Intuitionistic mathematics is a mental construction, essentially independent of language" 13 ,

"Thus for a human mind equipped with an unlimited memory, pure mathematics, practised in solitude and without using linguistic signs, would be exact, but the exactness would be lost in mathematical communication between human beings with an unlimited memory, because they would still be thrown upon language as their means of understanding." ${ }^{14}$ "First act of Intuitionism completely separates mathematics from mathematical language, in particular from the phenomena of language which are described by theoretical logic, and recognizes that intuitionist mathematics

\footnotetext{
${ }_{12}$ Brouwer [1908], p. 108.

${ }^{13}$ Brouwer [1947], p. 477.

14 Brouwer [1933], p. 443.
} 
is an essentially languageless activity of the mind having its origin in the perception of a move of time, i.e. of the falling apart of a life moment into two distict things, one of which gives way to the other, but retained by memory." 15

I would like to note here that Brouwerian solipsism consists of two different components. His description of the formation (or generation) of the subject of mathematical activities is obviously outright solipsistic. It never refers to other persons nor any item of the external world. This is the first component. And the second component is his rejection of linguistic communication in general. The second group contains such claims about the impossibility of linguistic communication. But with regard to the latter component, it appears that Brouwer sometime takes a conciliatory attitude and adds some restrictions such as 'essentially' or 'in particular from the phenomena of language which are described by theoretical logic'. It seems that he permits some possibility of linguistic communication as an inessential or auxiliary means of communication in such situations. But on the other hand he says,

"In default of a plurality of mind, there is no exchange of thought either. Thoughts are inseparably bound up with the subject." 16

Therefore, it seems that there is no room for the reading of Brouwer as a person who primarily rejects linguistic comunication but in some cases permits it as an auxiliary means for communication. It is true that he is a kind of solipsist and that he also rejects the possibility of linguistic communication completely. But I think it is important what the word 'linguistic' in linguistic communication means. With a particular interpretation of 'linguistic', I would like to insist that Brouwer does not completely reject the possibility of communication in general. In the following I will argue this point.

In the proceeding quotation, Brouwer strongly rejects the possibility of linguistic communication. Before that he presents an argument which refutes the existence of other minds. In Brouwer's philosophy, the notion of causal attention plays an important role. It plays the essentiall role of creating an external world including other individuals from sensations.

Causal attention repeatedly leads to identification of sensation complexes originating from causal acts of the subject, with sensation complexes experienced in causal connection with other individuals. On account of

15 Brouwer [1952], pp. 509-510.

${ }^{16}$ Brouwer [1948], p. 485. 
this identification the latter sensation complexes are called acts of those other individuals. ${ }^{17}$

Other individuals emerge to the subject through a certain resistance from the part of the objects as sensation complexes. However, those other individuals are not yet other minds. It is important that Brouwer thought that the transition from object individuals to other minds was made possible by the use of 'civilized language'. According to my interpretation of Brouwer's words, to causal attention of the subject, actions of the object individuals appear to be the same in quality as those of the subject individual. And this homogeneity of subject individual and object individuals makes it possible to ascribe minds to object individuals. But, as Brouwer argues, we cannot maintain this homogeneity for them. For let us suppose the subject ascribes minds $M_{1}, M_{2}, \ldots, M_{n}$ to object individuals $I_{1}, I_{2}, \ldots, I_{n}$. By ascribing such minds, the subject mind must elevate itself to a mind of the second order. I think this is because the subject mind would again experience minds of the second order as new sensation complexes. In consequence, the subject must assign a mind of the second order to every individuals once more, so this kind of assignment will continue ad infinitum. Metaphorically speaking, we could never erase the peculiarity of the subject as a fixed point of assignment of minds to individuals. So we cannot secure other minds as homogenious to the subject mind ${ }^{18}$. This is Brouwer's argument against the existence of other minds.

However, it is obvious that Brouwer's above argument for the non-existence of other minds is not successful. He refutes only the homogeneity of the subject mind and other minds. The non-existence of other minds does not follow immediately from the heterogeneity of the subject mind and other minds. But my main concern here is not to evaluate whether his argument is successful or not. My point is that the notion of mind is just a linguistically biased notion or linguistically constructed notion in Brouwer's philosophy. It seems that he argues for the impossibility of linguistic communication because of a failure of securing the existence of other minds. But such failure was built-in to the notion of minds from the beginning. In fact, we can find in his text that he uses another word 'soul' and with regards to it he basically admits the possibility of arriving at mutual understanding (with seveal restrictions). Why does he prohibit the former notion from playing the role which is permitted for the latter one? My answer is that it is because the notion of mind is coined in the system of language. If this interpretation is correct, it turns

17 Brouwer [1948], p. 482 .

18 Brouwer said that if we ascribed not minds but 'reason' to those individuals in order to characterize their behaviour, such elevation would not come. I think this is because the word 'reason' would apply to many other things, for example, mechanical things or animals and such applicatons of the word would not give raise to a homogeneity. 
out that his hostility to language precedes his hostility to mind in his philosophy as a whole. Thus, although Brouwer had solipsist tendencies from the beginning of his career, he had to argue for the unreliability of language before he presented a solipsist manifesto. Then we are forced to ask what is Brouwer's conception of language which makes criticism of logicist and formalist mathematics and fabrication of mind possible. I would like to investigate this in the next section.

\section{Brouwer's Conception of Language}

Brouwer [1929] which is titled 'Mathematics, Science, and Language' occupies a special position in his writings. It is not only because the paper is one of few papers whose subject concerns language and society, but also because it strongly reflects his activity as a member of Signific Circle. Although I will not mention to the details of Signific Circle, I would like to note that the purpose of the organization is to realize social reform through improvement of language. If Brouwer's activity in Signific Circle would be as such, as a matter of course he must presuppose that language or language use is able to have some effect on individuals as members of a society. Concretely speaking, what Brouwer had in mind was the fact that political propaganda against certain foreign countries stired the nation's feelings of hostility toward those countries in World War I. Brouwer was prompted to investigate the mechanism of how words can move people's feelings. Without this background we cannot understand Brouwer [1929] properly ${ }^{19}$.

${ }^{19}$ On the details of Signific Circle, see van Dalen [1999]. The following quotation from it is valuable for understanding the background of Brouwer [1929].

The suppression of one's fellow man by means of private property and trade would be impossible without words-of-power, such as enterpreneur, interest rate, profit. And the morphinizing of the conscience, required for the perpetuation of social injustice would be impossible without words-of-power like happiness, religion, art, civilization, genius, duty, reliability. These means of defence of injustice, among which the word fatherland is one of the most powerful, can only be destroyed by ending the present anarchy in the formation of words. And thus there emerges the primary social task, imposed on us by our conscience: the founding of an institute for language-reflection that first will have to brand the words-for-power of injustice in extensive analytical manifestos, and that subsequently will have to occupy itself with the forming of words for the realization of the common intuitive sense of justice, as soon as the eugenetics for this sense of justice will have created a possibility for development. (van Dalen [1999], p. 249.)

Here we can find an expression of Brouwer's intention of fighting social injustice and the purpose of organizing Signific Circle. Another thing that we can see in the quotation is that the pattern of Brouwer's action for fighting social injustice is very similar to the pattern of the first act of intuitionism. 
In this paper Brouwer explains in detail how groups or organizations which vary in size are organized, maintained and controlled. We need not enter into an explanation here, but I would like to touch on two important points.

One is that the intuition which underlies mathematical constructions also supports our recognition of the outer world. In Brouwer's philosophy it seems that there is no problem concerning the applicability of mathematics to our actual world. For our objective world, which consists of various objects and events to which mathematics is applied, is itself constructed by the same intuition as the one which makes mathematical constructions possible. According to Brouwer everything which includes mathematics is generated from the chaotic state of conciousness by a kind of ability (or an act of will) called Mathematical Attention. He divides this Mathematical Attention into three sub-abilities called time awareness, causal attention and mathematical abstraction respectively. Time awareness, which is also called the intuition of two-oneness (or two-ity), is an act of will which makes possible the act of the 'falling apart of a moment of life into two qualitatively different things of which one is experienced as giving way to the other and yet is retaind by an act of memory'. In this way, on the one hand, time awareness generates a natural number sequence and, on the other hand, it generates a time sequence of phenomena which is the basis of the perceptual world. With regards to the perceptual world, causal attention plays an essential role which identifies different time sequences of phenomena and, for example, constructs an object as an equivalent class of sequences of phenomena modulo causal connection so to speak. On the other hand, with regards to mathematics, mathematical abstraction plays an indespensable role in constructing a mathematical system. Its role is to strip twoity of its material contents and to retain it as an empty form which is the common substratum of all two-ities. This common substratum is called the Primodial Intuition of Mathematics. And, as Brouwer says, 'the effectiveness of mathematical abstraction is based on the fact that many causal sequences are much easier to control if they are projected on subsystems of pure mathematics, that is, if their empty abstractions are embedded in more extended pure mathematics'. It is important to notice here that in this way there is no problem about the applicability of mathematics in Brouwer's philosophy.

The second point to be noted is that both scientific theories (that is, causal sequences that are embedded in mathematics and made easier to control) and pure mathematics are 'hypotheses' for Brouwer to the effect that both are constructed by an act of will. In particular, I would like to point out that the perceptual world itself is constructed by an act of will and therefore it is just a hypothesis.

Keeping Brouwer's above view on the world and mathematics in mind, I would like to focus on his view of language in Brouwer [1929]. When he analyzes the effect of language on members of a society, the conception of language which he 
presents is an outright communication-based one. According to his views, language is nothing but a means for will-transmission. In this view of language he describes how will-transmission developes from the primitive stages of human society to a civilized society in a process of complexification of human societies. In order that it is possible for him to reform a society through an improvement of language, it must be presupposed that there is a certain linguistic mechanism which makes it possible to maintain and control certain groups or organizations. He describes such a mechanism as follows,

On the other hand in every-life there is no need for stability and exactness of language, collective will and automatism-of-training have made people 'good understanders'.

Even if language originally and in the first place is a function of activity of social man, it has some significance in the processes of reflection and mnemotechnique in the solitude of individual man, partly because of habitautomatism following the use of language, partly because of the role which science and social organization continue to play even in the thought-world of solitude ${ }^{20}$.

Thus it turns out that language is merely an instrument of will-transmission which makes human social activities possible. This claim may sound atypical in light of the usual image of Brouwer, but such an impression is incorrect. It is true that phrases which suggest his solipsist tendencies are scattered throughout his writings and it is unmistakable that he has such solipsist tendency from the beginning. But when he writes in particular about the non-existence of other mind and the impossibility of mutual understanding he does so very cautiously, that is, he does so only according to the particular conception of language ${ }^{21}$. Then what is this particular conception of language? Brouwer writes,

In other words, the practical reliability of the logical principles is based on the fact that most of the perceptual world in its finite organization displays much greater trust and contentment than mankind itself. Man has for ages

${ }^{20}$ Brouwer [1933], pp. 422-443. Brouwer also writes in Brouwer [1929], p. 48.: 'Language, therfore, is altogether a function of the activity of social man. That individual man in solitude uses language as an aid to memory is only due to the fact that in his thinking science and the organization of society have to be taken into account. And if language accompanies processes that are transcendent and do not involve action, it is only because the whole of human activity is subjected to the transcendent influence of the free will.'

${ }^{21}$ We might not insist this about all his writings. 
been blind to this simple interpretation because he did not recognize that words in essence are nothing but an instrument of will transmission, and in foolish superstition started to treat words as labels of some fetish-like "concept." 22

As is clear from this quotation, Brouwer insists not only that the application of logical principles to infinite domain is a mistake. But rather he calls the conception of language itself, which produces such a mistake, into question. Let us call this conception of language, according to which we treat words as labels of some concept, as concept-oriented language. So Brouwer does not criticize the notion of language in general, but he rejects the notion of concept-oriented language. We can see the reasoning for his rejection of it in his picture of the spatio-temporal world. In his philosophy the notion of a spatio-temporal world inhabited by objects is just a hypothesis which is constructed by an act of will. Then the notion of concept which is formed from such hypothetical objects must be hypothetical in itself. Even if we could describe the structure of mathematical constructions by using certain concepts, such a procedure would contravene the Brouwerian point of view. Therefore, it is clear that Brouwer presents two conceptions of language here and make a comparison between them. One is the view that language is nothing but an instrument of will-transmission. The other is the notion of concept-oriented language which makes semantic projects possible as well as logicist and formalist projects. Brouwer holds the former conception of language so that he can engage in the project of Signific Circle. This is obvious from the fact that he analizes the effects of words on individual members of a society only in terms of the dynamics of will-transmission. And Brouwer rejects the latter conception of language completely because the latter one is a deviation from the former one, that is, from the genuine and original notion of language. The excessive productivity of language as we mentioned earlier is just a feature which is brought about by such a deviation ${ }^{23}$.

\section{Concluding Remark}

We have seen that Brouwer's criticism of logicist and formalist mathematics was

${ }^{22}$ Brouwer [1929], p. 49.

${ }^{23}$ As is well known, Brouwer [1929] is the manuscript of "First Guest Lecture" given in Vienna on March 10, 1928 and Wittgenstein attended it. In evaluating Brouwer's influence on Wittgenstein, it was an issue so far whether Brouwer's lecture caused Wittgenstein's conversion to constructivism. But as recent research makes it clear, if Wittgenstein already had a constructive tendency, the issue would not be meaningful. However, if one of the significant points of his lecture is to compare two conceptions of language, another possibility concerning Brouwer's influence on Wittgenstein would emerge. 
based on his criticism against the excessive productivity of language. Then, however, a question about the notion of content may arise from this interpretation. As we saw, language is primarily a means of will-transmission for Brouwer. But if language is such a means, then it seems that it must be also a means of transmitting content of the will. If Brouwer would reject the concept-oriented notion of language, then how could he preserve the notion of (propositional) content?

One answer to this question is as follows. It is true that Brouwer rejected the notion of concept and propositional function thoroughly, but at the same time he did not also completely reject the notion of propositional content by rejecting them. Rather, what he rejected was, in fact, to detach the content of a proposition from the method of arriving at the content. In the case of mathematics, this means that we cannot separate the content of a mathematical proposition from its proof.

According to this interpretation, Brouwer's rejection of the concept-oriented notion of language amounts to his rejection of the detachbility of content and proof. I think this reading of Brouwer is true as long as we stick to maintaining a semantically tractable notion of content. And by taking the requirement of the undetachbility of content and proof as a constraint for admissible propositional functions, we can avoid the chimerical 'everything' as our domain. Also, there are some passages which suggest that Hilbert interpreted Brouwer's criticism of classical mathematics as the requirement of such an undetachbility ${ }^{24}$.

${ }^{24}$ For example, in Hilbert [1922], although he severely criticizes Brouwer-Wyle project of reformation of mathemtics, his analysis of the failure of the logicist project has substantialy the same content as that of Brouwer's. Hilbert writes;

Frege tried to ground number theory on pure logic, Dedekind tried to ground it on set theory as a chapter of pure logic : both failed to reach their goal. Frege did not treat carefully enough the usual concept-formations of logic in thier application to mathematics: so he held the scope of a concept to be something immediately given, and he believed he was entitled to take these scopes themselves unrestrictedly as things. He thus fell to some extent into an extreme realism of concepts. (Hilbert [1922], pp. 201-202.)

On the other hand, he also writes concerning an interpretation of general statements like $x+y=y+x$,

Such an equation ... is not viewed as a claim for all numbers. Rather it is interpreted in such a way that its full meaning is given by a proof procedure: each step of the procedure is an action that can be completely exhibited and that follows fixed rules.

This consideration helps us to gain an understanding for the sense of the paradoxical claim, made recently by Brouwer, that for infinite systems the law of the excluded middle (the "teritium non datur") loses its validity. (these two passage from Hilbert's lecture in winter term 1920 are quoted from Sieg [1999] pp. 25-26.) 
But I would like to insist that another ansewer is also possible. Brouwer emphasized the notion of language as a means of will-transmission and as a function of the activities of social man. This is nothing but emphasis on the dynamical aspects of language. And I think the emphasis on these aspects of langauge means that Brouwer's conception of language is not just that of an exchange of contents but that of a kind of fabric of actions which suggests something analogus to the notions of language game. In this case, a content of the will must be read off from the role which the subject's particular utterance or action plays in such a fabric of actions. Perhaps such a notion of content may be too coarse to use as a semantically tractable one $^{25}$. But given Brouwer's thorough rejection of the concept-oriented notion of language, I think this interpretation is more congenial to Brouwer's overall philosophy than the former interpretation. We can also see the novelty of Brouwer's thought in that he contrasts two completely different conceptions of langauge and he uses such a contrast in order to descriminate between the linguistic and the mathematical.

\section{References}

Brouwer, L.E.J. [1907] "On the Foundations of Mathematics", in Brouwer [1975] pp. 13101.

_ [1908] "The unreliability of the logical principles", in Brouwer [1975] pp. 107111.

[1912] "Formalism and Intuitionism", in Brouwer [1975] pp. 123-138.

[1929] "Mathematik, Wissenschaft und Sprache", in Brouwer [1975] pp. 417-428.

("Mathematics, Science and Language", English translation in Mancosu [1998])

- [1930] "The Structure of the Continuum", in Mancosu [1998] pp. 54-63.

[1933] "Will, Knowledge and Speech", English translation of "Willen, Weten, Spreken", in van Stigt [1990], pp. 418-431.

[1975] Collected Works vol.1 North-Holland.

van Dalen, D. [1999] Mystic, Geometer, and Intuitionist: The Life of L.E.J. Brouwer, Oxford, 1999.

Detlefsen, M. [1990] "Brouwerian Intuitionism", Mind 99.

Frege, G. [1924/25] "Erkenntnisquellen der Mathematik und Naturwissenschaften", in Nachgelassene Schriften, 1969.

Hilbert, D. [1900] "On the concept of number", in Ewald, From Kant to Hilbert, vol. 2 1996.

- [1922] "The New Grounding of Mathematics : First Report", in Mancosu [1998], pp. 198-214.

Mancosu, P. [1998] From Brouwer to Hilbert, Oxford, 1998.

Sieg, W. [1999] "Hilbert's Programs: 1917-1922", in The Bulletin of Symbolic Logic, vol. 5, No. 1, March, 1999.

${ }^{25}$ In the case of mathematics, such a role played by a mathematical proposition may be its contribution to the overall proof. Then it is possible that these two interpretation of Brouwer's conception of language would coincide with each other. 
van Stigt, W. P. [1990] Brouwer's Intuitionism, North-Holland.

[1979] "The Rejected Parts of Brouwer's Dissertation on the Foundations of Mathematics", in Historia Mathematica, vol. 6, No. 4, pp. 385-404. 